\title{
The Role of Advanced Distribution Automation in Smart Grid
}

\author{
Priyanshu Sachdeva \\ Dept. of Electrical and Electronics \\ Engineering, \\ Manipal Institute of Technology \\ Manipal, India
}

\begin{abstract}
The Advanced Distribution Automation System is a vital part of the Smart Grid initiative, and also a reliable communication network that constitutes the core of the Automation Systems [6]. Self-healing for smart distribution network is based Advanced Distribution Automation (ADA) and is one of the key core function of the smart distribution network. ADA gives us additional benefit of dealing with threats which maybe internal or external to the power system [3]. This way efficiency and effectiveness of the grid can be increased.
\end{abstract}

Keywords-Smart Grid, Distribution Automation, Advanced Distribution Automation, Self-healing, Distributed Substation Control.

\section{INTRODUCTION}

Smart Grids are enabling technologies aiming to resolve major

challenges arising from the aging utility infrastructure, rising energy demands, and growing concerns over the excessive use of exhaustible resources such as carbonbased fossil fuels for energy generation. In order to build intelligent features into the existing traditional power grid, a comprehensive technological approach compromising of real-

time monitoring systems, decision-making algorithms, control systems, forecasting and optimized algorithms are essential. For implementing this technologies in smart grids must include: Distribution Automation (DA), asset management, Advanced Metering Infrastructure (AMI), renewable energy resources. In simultaneousness with the deployment of information technology and telecommunication networks, smart grids now allows the utility companies to monitor and optimize the production and distribution of power in nearreal time. This is possible because of the smart power generation that allows and involves the two way flow of electricity and information. The generation is based on the consumption or the expected demand and the demand can drive the generation as or when it desires.

The term Advanced Distribution Automation (ADA) can be put up as the automation of all the features related to the distribution system using the information that has been collected from various sub-stations, devices deployed on the grids and the smart meters at the end location. The most important aspects while designing an effective distribution automation system is protection and switching functions. Nowadays, various DA devices have been deployed in the distribution lines to track current and voltage state at various times, to exchange device information and to reconfigure the network to meet the regular changes in the environment.

SCADA (Supervisory Control And Data Acquisition) that monitors and controls the various distribution substations is considered as Advanced Distribution Automation. This system provides an extra benefit of remotely controlling and observing the renewable energy sources (RES). The SCADA system monitors and can make slight changes in the system to function properly. This system is a closed loop system and works with very less human intervention and also has the ability to monitor the entire system in near-real time.

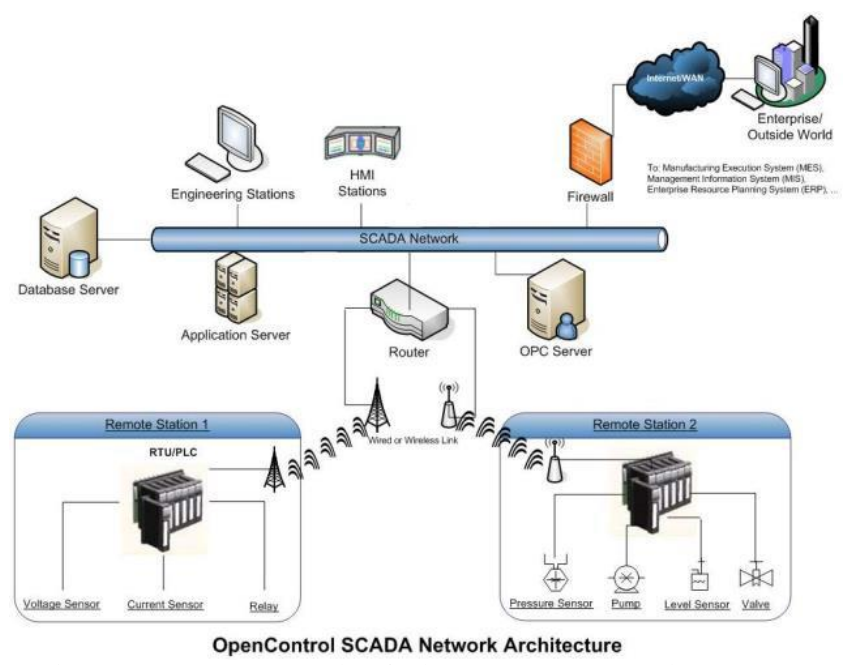

Figure 1: Open Control of the SCADA Network Architecture.

\section{APPLICATIONS OF ADVANCED DISTRIBUTION}

AUTOMATION IN SMART GRID

The most important application of the Advanced Distribution Automation is fault diagnosis by monitoring the faults in the grid, then identifying the root cause of the occurred fault and then restoring the system. Automated fault and root-cause identification can also been investigated using the state of art of technologies such as cause-effect network, Artificial Intelligence or Bayesian inference. The deployment of wireless 
sensors, distributed actuators, and the present day information and communication technologies would generate more accurate real-time data about the states, making automated fault diagnosis feasible and applicable in future intelligent smart grids. The application of the Advanced

\section{Integrated Volt-Self-Var Control (IVVC) \\ 3. Equipment health monitoring \\ 4. Fault Location, Isolation and Service Restoration} (FLISR) systems

5. Integration of Renewable Energy Sources.

\section{DISTRIBUTION AUTOMATION}

Distribution Automation technologies and systems can achieve substantial grid impacts and benefits:

1) Improvement in location of fault, isolation, and service restoration capabilities that result in less number of outages, less operational and maintenance costs, less chances of equipment failure or damage, and lesser inconvenience at the consumer end.

2) Improved distribution system resilience to extreme weather events by automatically limiting the extent of major outages and improving operator ability to diagnose and repair damaged equipment.

3) More effective equipment monitoring and preventative maintenance that reduces operating costs, enables more efficient use of capital assets, reduces the likelihood of equipment failures.

4) More efficient use of repair crews and truck rolls that reduces operating costs, enables faster service restoration, and lowers environmental emissions.

5) Improved grid integration of selected distributed energy resources (DER) such as thermal storage for commercial and municipal buildings.

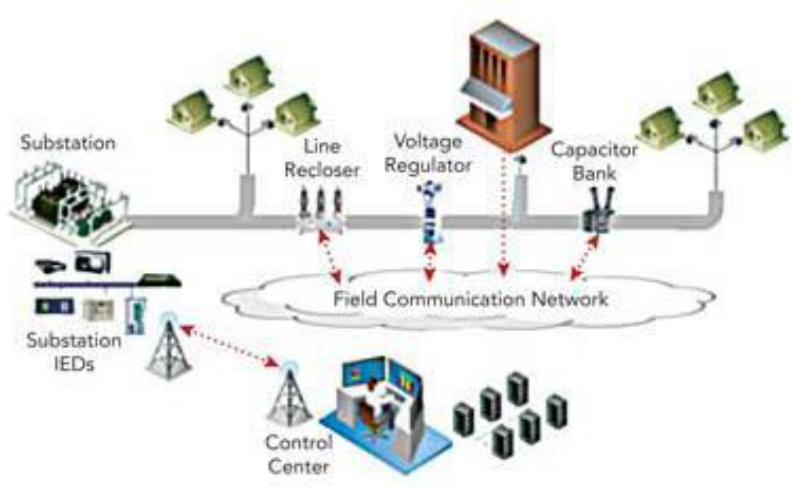

Figure 2: Illustration of Distribution Automation system Distribution Automation Field Devices includes:

\section{Remote Fault Indicators}

These are sensors that detect when current and voltage levels on feeders are outside the threshold boundaries. Operators can use this data to rapidly determine the location of the fault (such as an equipment failure) or
Distribution Automation can be grouped into 5 major subgroups:
1. SCADA (Supervisory
Control
And Data Acquisition) System.

distinguish between a fault and temporary high load demands.

\section{Smart Relays \\ Smart relays apply sophisticated software to accurately detect, isolate, and diagnose the cause of faults. They may be installed at substations for feeder protection or on devices in automated switching schemes. Device controls are activated according to equipment settings and algorithms.}

The relays also store and process data to send back to grid operators for further analysis.

\section{Automated Feeder Switches and Reclosers}

Automated feeder switches open and close to isolate the faults and repair the feeder with the fault to restore power back to customers on line segments without a fault. They are typically configured to work with smart relays to operate in response to commands from an autonomous control system, distribution management systems, and signals from grid operators.

Switches can be configured to close and open at predetermined intervals when fault currents are detected. This action is called as reclosing of the switches, and are deployed to sectionalize faulty sections of the feeders and to divert power around the faulty section of the feeder and re- energize only after the obstruction has been cleared by itself from the feeder line.

\section{Automated Capacitors}

Utilities use capacitive bank (made up of capacitors) to compensate for reactive power requirements caused by inductive loads from customer equipment's, transformers, or overhead lines impedances. Compensating for reactive

power reduces the total amount of power that need to be provided by power plants, resulting in a flat voltage along

the feeder, and less energy being loosed as electrical losses in the feeder line.

Automated Voltage Regulators and Load Tap Changers Voltage regulators are types of transformers that make small adjustments to voltage levels in response to changes in load. They are installed in substations. At the sub-stations these transformers are called as load tap changers and along distribution feeders to regulate downstream voltage.

\section{Automated Feeder Monitors}

Feeder monitors measure the load on distribution lines and equipment and can trigger alarms when equipment or line loadings start to approach potentially damaging levels.

Monitors deliver data in real time back to the systems 
so

that utilities can efficiently assess the change in load trends and take corresponding actions, such as taking equipment off service, transferring of load on substitute feeder, or repairing equipment whenever necessary without causing any power outage.

\section{FAULT LOCATION, ISOLATION AND SERVICE RESTORATION (FLISR) SYSTEM}

Distribution Automation provides advanced features for operators to detect, locate, and clear the faults in real time. Relay switches, remotely placed fault indicators, and reclosers provide access to real-time data on the feeders, which when delivered to a functional Distribution Management System (DMS), can help us accurately figure out the root cause of the fault and location of faults and the extent of fault when they occur. This technology involves automated feeder switches and reclosers, communication networks, grid analytics [1]. These technologies go hand in hand to regulate power restoration by automatically isolating faults that have been occurred on the feeder and restoring the service to remaining customers by transferring them to nearby sub-station feeders via other distribution lines. This reduces the number of customers affected by the outage caused by the fault and the length of fault interruption. Automated actions usually requires less than a minute when compared to traditional manually validated FLISR actions that usually require five minutes or more [1].

Precise fault location enables the operators to send the maintenance work force on the right, précised location and notify customers of the status of power being outage, which reduces mmaintenance costs, reduces the burden on customers, and help in maintaining the consumer satisfaction [1].

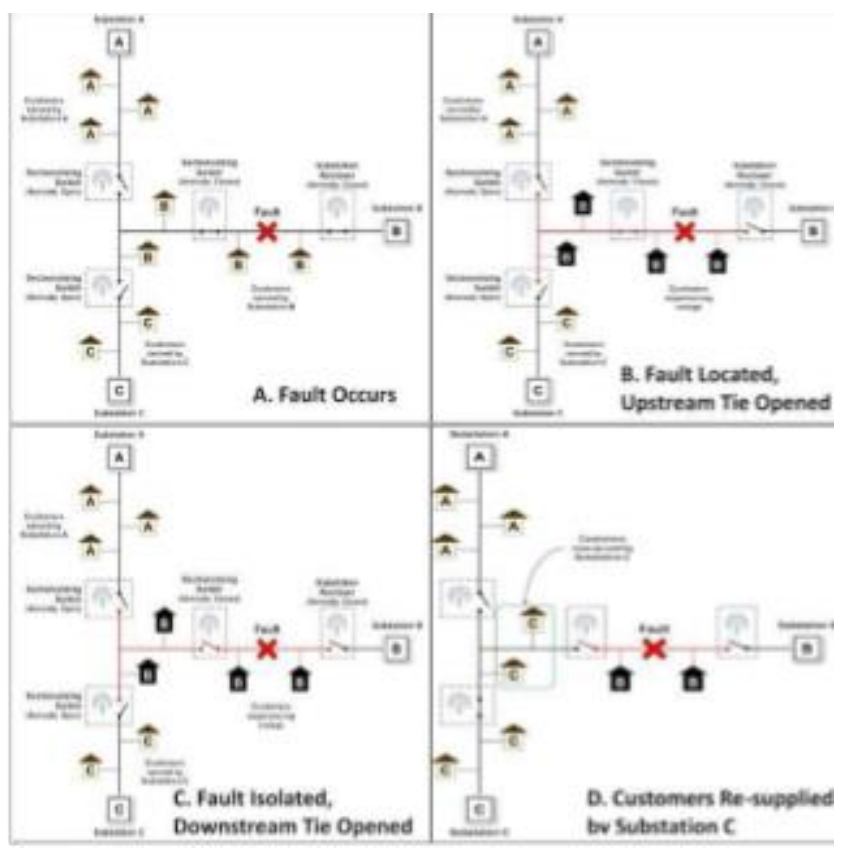

Figure 3: Illustration of FLISR Operation.
In (Figure 3-A), When a fault occurs, the FLISR system locates the fault

In (Figure 3-B, 3-C): Once the fault is located, the system isolated the feeder on both sides of the fault from both the ends Feeder is re-energizes and that portion of the feeder with the fault cleared now supply power to all customers served by those feeder sections from another (Figure 3-D)[1].

The fault isolation feature of this technology help's the repair \& maintenance crews to precisely locate the trouble spots, resulting in shorter outage durations for the customers.

\section{SCADA (SUPERVISORY CONTROL AND DATA AQUISITION)}

SCADA is a legacy system for many utilities, responsible for monitoring and control of substation relays, distribution switches, and other equipment and sensor points fromthroughout the electric distribution system to ensure that voltages and currents are managed within operational requirement When integrated with other DA systems, it provides a platform for a range of distribution management functions. SCADA systems process operational data from the field and displays status on control center operator screens or the HMI (Human Machine Interface). Abnormal conditions trigger display alarms, which are then acted on by the grid operators that can issue operator commands.

DMS can also automatically issue SCADA commands.

Locating faults using SCADA allows utilities to isolate and repair faults and restore service quickly, thereby increasing system resilience. SCADA equipment at the transmission and substation levels is used when the system conditions require load curtailments in ways that balance system protections with the varying consumer needs. SCADA technologies are also be used to facilitate monitoring of customer-owned generation to enhance safety and improve energy management. SCADA software and master stations, with proper switching and reclosers, can identify access points for customer loads and allow for real-time monitoring of all types of distributed generation assets, including back- up generators.

SCADA integration typically involves interfaces with a variety of systems and sources of grid information,

including:

- GIS for distribution network models

- Real-time operating and switching information from DMS

- Measurements and data to DMS and data historians

- Measurements and data from MDMS, or data warehouses.

When interoperable with other systems (i.e., DMS, 
MDMS, OMS, CIS, GIS, and DERMS), SCADA provides:

- Distribution control for substations, feeders, inter-tie points, and distribution equipment

- Sequencing of events, time-stamped data, trending, and diagnostic data for load management, outage analysis, and demand response

- Web-based access for operators, engineers, and managers for accomplishing system operations, outage management, and operations and maintenance activities every hour of every day throughout the year.

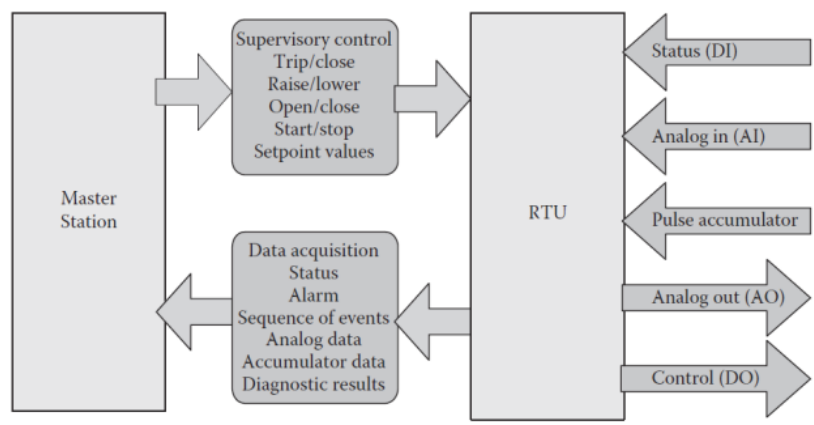

Figure 4: SCADA system data flow architecture.

\section{INTEGRATED VOLT-VAR CONTROL}

Reactive power flow and feeder voltage are very closely related and dependent variables. Any control action to change any of the variable results in opposing the other one, in case of a scenario where by raising the voltage using step up transformer at the substation end can produce a rise in voltage across the feeder and could cause the capacitor bank control system to remove the capacitive bank from functioning, thus reducing the voltage across the feeder. Placing a capacitive bank at a substation will decrease the voltage at the substation [2].

Automated regulation of voltage and power factor correction enables utilities to reduce peak demands, utilize the present assets more effectively and efficiently, and improve power quality for the growing digital economy.

Automated power factor correction provides grid operators with new capabilities for managing reactive power flows and boosting the overall power quality.

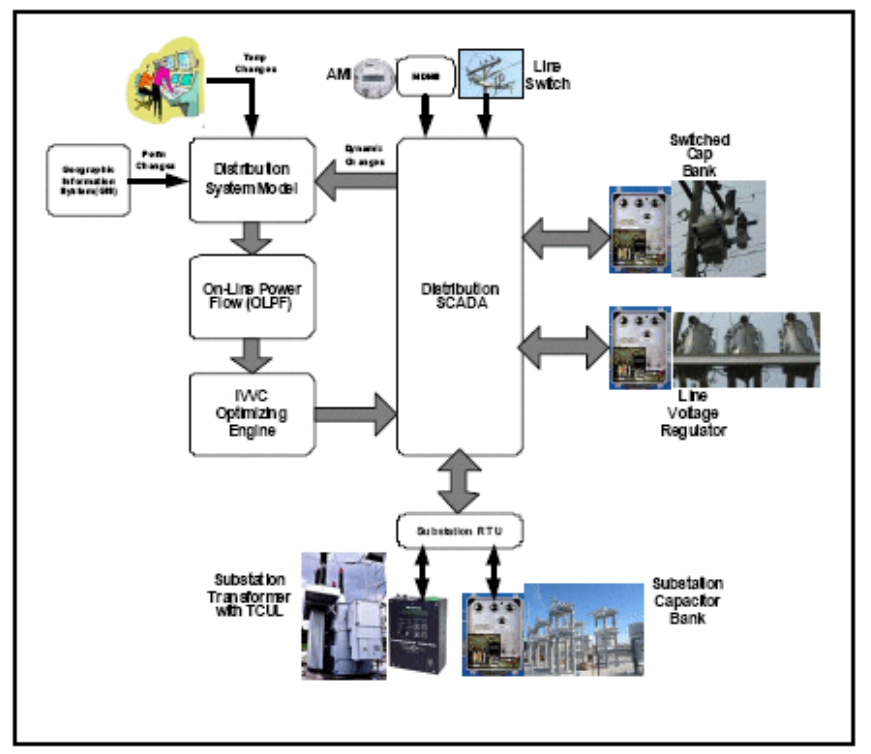

Figure 5: A typical Integrated Volt-Var Control system.

\section{EQUIPMENT HEALTH MONITORING}

Installing sensors on key electrical machinery and infrastructures such as distribution lines to measure the health of the equipment and can provide nearreal time alerts for any unusual circumstances as well as analytics that help maintenance engineers plan equipment maintenance, repair, and replacement. This technologies also equips the grid operators with better understanding to send the maintenance crews relying on the realtime data that has been diagnosed for the system. Several utilities automate monitoring systems to reduce human interventions during inspections, enable active maintenance, and better diagnose equipment failures so it can rectified and bought back into service to prevent any outages.

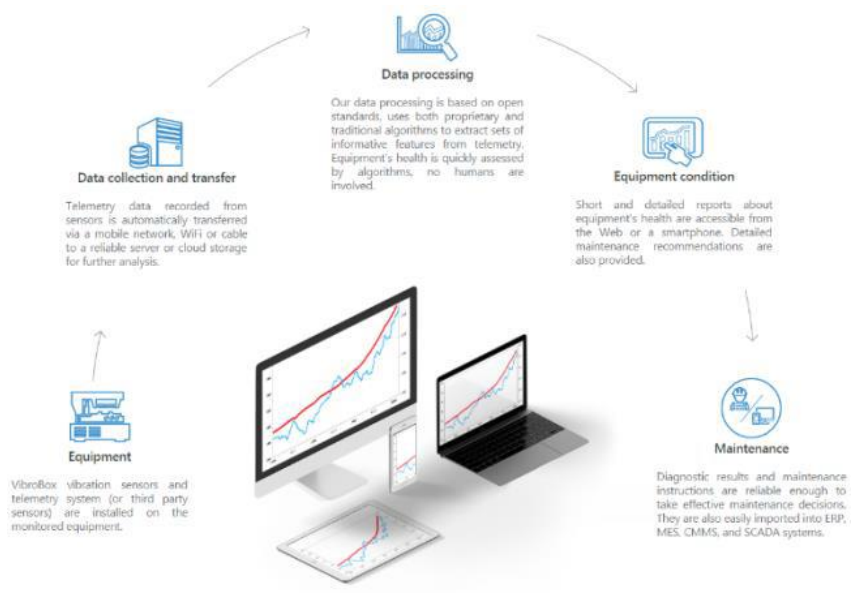

Figure 6: Equipment Health Monitoring System 


\section{INTEGRATION OF DISTRIBUTED ENERGY RESOURCES (DER)}

Integration of DER in the smart grid requires state of the art tools to monitor and use the distributed energy resources to address control issues and overall flow of power, such as requirement of low voltage, voltage fluctuations, harmonic injection and proper management of reactive power. Some smart grid utilities have started to implement "Distributed Energy Resource Management Systems" (DERMS) and "Integrated Automated Dispatch Systems" (IADS) on small scale installments. Various utilities have already started connecting thermal energy storage for industrial buildings.

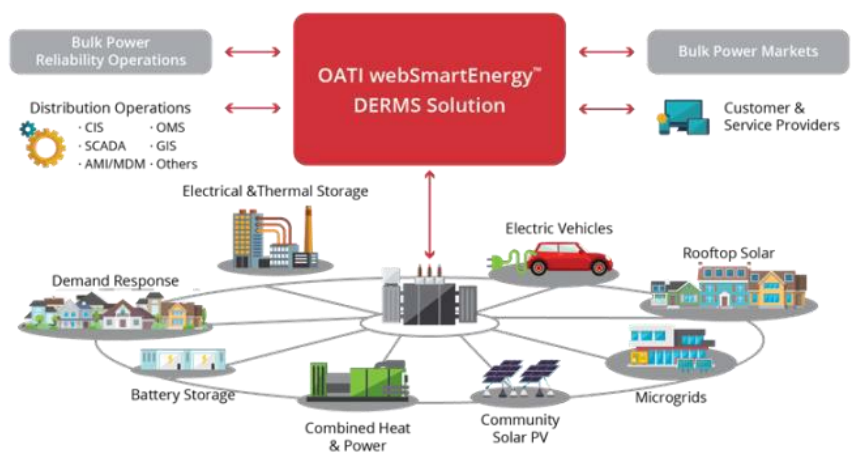

Figure 7: Integration of Distributed Energy Sources

\section{SUMMARY}

ADA plays a crucial part in the implementation of the smart

grid objectives,

1) Self-Healing control (FLISR)

2) Equipment Health Monitoring

3) Efficiency improvement

\section{REFERENCES}

[1] Retrieved from www.smartgrid.gov

[2] R W Uluski. "The role of Advanced Distribution Automation in the Smart Grid", IEEE PES General Meeting, 2010

[3] Wei Liu, Taifeng Kang, Wenxin Cheng, Fengqing Zhao. "The modeling of self-healing control system for distribution network based on UML", 2015 5th International Conference on Electric Utility Deregulation and Restructuring and Power Technologies (DRPT), 2015

[4] https://new.siemens.com/global/en/products/energy/topics/connecting - grids.html

[5] https://new.siemens.com/global/en/products/energy/energyautomation- and-smart-grid/grid-applications/self-optimizinggrid.html

[6] N. Nakhodchi, S. Alishahi, M. H. Pourarab, Guohong Wu. "Result assessment of the first local micro-grid in Mashhad, Iran", 2015 IEEE International Telecommunications Energy 